\title{
Segurança e Eficácia da Reutilização de Materiais para Angioplastia Transluminal Coronária Percutânea: Estudo REMCLI-ATC
}

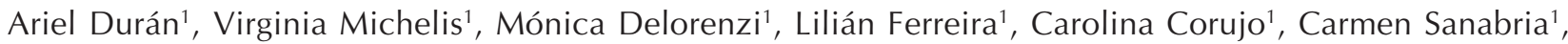 \\ Isabel Guevara ${ }^{1}$, Aurora Cioli ${ }^{1}$, Orieta Rivero' ${ }^{1}$, Valeria Borja ${ }^{1}$, Mónica Natelli' ${ }^{1}$, Ricardo Lluberas ${ }^{1}$
}

\section{RESUMO}

Introdução: Apresentamos nossa experiência no estudo REMCLI-ATC (REutilización de Materiales no hospital das CLInicas-ATC), que foi desenvolvido no Setor de Hemodinâmica do Departamento de Cardiologia do Hospital de Clínicas da Faculdade de Medicina de Montevidéu, Uruguai, entre setembro de 2001 e agosto de 2002 e entre janeiro de 2003 e dezembro de 2004. Método: Foram incluídos, prospectivamente, 100 pacientes submetidos a angioplastia transluminal coronária (ATC), nos quais se utilizou aleatoriamente material novo (grupo $1, \mathrm{n}=49$ ) ou reesterilizado (grupo $2, \mathrm{n}=51$ ), analisando-se uma série de variáveis que avaliaram a eficácia e a segurança de cada um dos grupos. Resultados: O sucesso clínico e angiográfico no grupo 1 foi de $100 \%$ e no grupo 2, de $96 \%(p=0,54)$. Não foram observadas diferenças em relação ao número de cateteres-guia, guias e balões utilizados na ATC entre os grupos. O volume de contraste e os tempos de radioscopia e do procedimento também não apresentaram diferenças. As complicações maiores ocorreram somente no grupo $2(0 \%$ vs. $3,9 \% ; p=0,17)$ e as menores ocorreram em ambos os grupos $(6,1 \%$ e $9,8 \%$, respectivamente; $p=0,48)$. Conclusão: Concluímos, portanto, que é tecnicamente factível e seguro utilizar material reesterilizado nas ATCs, o que permitiria, uma economia considerável para países como o Uruguai, com recursos econômicos limitados.

DESCRITORES: Angioplastia transluminal percutânea coronária. Reutilização de equipamento. Equipamentos descartáveis/economia. Segurança de equipamentos. Custos hospitalares. Redução de custos.
1 Setor de Hemodinâmica - Departamento de Cardiologia do Hospital de Clínicas - Faculdade de Medicina da Universidade da República - Montevidéu, Uruguai.

Correspondência: Ariel Durán. Av. Italia, s/n - Hospital de Clínicas de la Facultad de Medicina de Montevideo - 1er piso - Departamento de Cardiología, Sector Hemodinamia - Montevideo, Uruguay - CP 11300

E-mail: aduran@hc.edu.uy

Recebido em: 19/5/2008 • Aceito em: 12/8/2008

\section{SUMMARY}

\section{Safety and Effectiveness of Reprocessed} PTCA Materials: The REMCLI-ATC Study

Background: We describe our experience in the REMCLIATC (REutilización de Materiales no hospital das CLInicasATC) study, which was developed at the Hemodynamics Section of the Department of Cardiology of the Hospital de Clínicas of the Medicine School of Montevideo, Uruguay, from September 2001 to August 2002 and from January 2003 to December 2004. Methods: A hundred patients were prospectively included and treated with Percutaneous Transluminal Coronary Angioplasty (PTCA) with new (group 1, $\mathrm{n}=49$ ) or reprocessed (group 2, $\mathrm{n}=51$ ) material. Both groups were evaluated through variables that tested safety and efficacy of each strategy. Results: The clinical and angiographic success rate was $96 \%$ with reprocessed material and $100 \%$ with new material $(p=0.54)$. No significant differences were found between the groups regarding the number of guide catheters, guides and balloons used in the PTCA. The volume of contrast medium, radioscopy times and procedure times were also similar. Major complications were seen only in group $2(0 \%$ vs. $3.9 \%, p=0.17)$, while minor complications occurred in both groups $(6.1 \%$ and $9.8 \%$, respectively; $\mathrm{p}=$ 0.48). Conclusion: Our data showed that it is technically possible and also safe to treat patients using reprocessed material in PTCA procedures. Theoretically this would result in an important resource saving in economically limited countries like Uruguay.

DESCRIPTORS: Angioplasty, transluminal, percutaneous coronary. Equipment reuse. Disposable equipment/economics. Equipment safety. Hospital costs. Costs savings.
A Cardiologia Intervencionista alcançou grande desenvolvimento nos últimos anos e o número de procedimentos percutâneos tem aumentado progressivamente em todo o mundo ${ }^{1-3}$.

Habitualmente, nos países desenvolvidos, os materiais utilizados na angioplastia transluminal coronária (ATC) são descartados após o final do procedimento. De fato, guias e balões são projetados para serem usados uma única vez. No entanto, alguns países estão 
acostumados a reesterilizar esses materiais para que possam ser utilizados novamente. Os motivos para isso são vários, mas, sem dúvida, o fator econômico é o mais importante é $^{4-7}$.

Os métodos de reesterilização usados com maior freqüência são o óxido de etileno e, em algumas ocasiões, anti-sépticos líquidos potentes. Apesar dos numerosos relatos informais que aprovam essa conduta, não é freqüente encontrar na literatura médica trabaIhos com metodologia científica adequada que sustentem ou desaprovem essa medida ${ }^{8-15}$.

A hipótese de nosso trabalho é avaliar se, com adequada técnica durante o procedimento, bons cuidados após o procedimento e reesterilização adequada por métodos padronizados, a reutilização de materiais para ATC é segura e eficaz para o paciente e para a equipe intervencionista. A segurança dessa estratégia foi estudada em função da taxa hospitalar de complicações maiores e menores relacionadas ao procedimento. A eficácia foi avaliada comparando-se o tempo total do procedimento e de radioscopia de cada exame, o volume de contraste administrado, o número de cateteres-guia, guias e balões utilizados por paciente, e se houve ou não necessidade de encaminhálo para o outro grupo de estudo (crossover).

\section{MÉTODO}

O estudo REMCLI-ATC (REutilización de Materiales no hospital das CLInicas-ATC) incluiu prospectivamente e de forma randomizada todos os pacientes encaminhados para ATC no Setor de Hemodinâmica do Departamento de Cardiologia do Hospital de Clínicas da Faculdade de Medicina de Montevidéu, Uruguai, entre setembro de 2001 e agosto de 2002 e entre janeiro de 2003 e dezembro de 2004, que se adequavam aos critérios de inclusão e exclusão e que assinaram o formulário de Consentimento Informado.

A randomização foi realizada com base em uma tabela de números aleatórios obtida no programa EPI INFO versão 5 . Os resultados foram calculados conforme o grupo original de distribuição (intenção de tratar).

\section{Critérios de inclusão}

- Todos os pacientes encaminhados para ATC no Setor de Hemodinâmica do Departamento de Cardiologia do Hospital de Clínicas no período referido.

- Pacientes de ambos os sexos.

- Pacientes com mais de 18 anos de idade, sem limite superior.

\section{Critérios de exclusão}

- Manifestações clínicas sugestivas de quadro infeccioso sistêmico no momento do exame.

- Infecção cutânea em áreas de punção braquial ou inguinal.
- Falta de consentimento escrito ou impossibilidade de acompanhamento do paciente.

- Paciente que tenha sido incluído previamente neste ou em outro estudo.

O procedimento foi realizado por dois operadores especializados da equipe médica permanente de nossa instituição. Após o ingresso do paciente no setor e depois da realização da cineangiocoronariografia e da constatação de que do ponto de vista angiográfico era indicada a ATC, avaliava-se se o paciente preenchia ou não os critérios de inclusão e exclusão. Em caso positivo, o paciente era informado verbalmente sobre o estudo e recebia um folheto que explicava em linguagem acessível os objetivos e a metodologia do trabalho. Caso aceitasse ser incluído, pedia-se ao paciente que assinasse o Consentimento Informado e a enfermeira-chefe da sala escolhia o material de acordo com o resultado da randomização.

Tanto o paciente como o médico que faria o acompanhamento clínico ignoravam o tipo de material utilizado. O cardiologista intervencionista, embora capaz de reconhecer o tipo de material, limitava-se a realizar a ATC com o material indicado pela randomização em todos os casos.

Morte cardíaca ou não-cardíaca relacionada diretamente à intervenção, infarto agudo do miocárdio nãofatal, sangramento interno ou externo com necessidade de transfusão, isquemia grave do membro abordado com necessidade de cirurgia vascular de emergência ou eletiva, e septicemia relacionada ao procedimento foram consideradas complicações maiores.

Dor persistente na zona de punção, hemorragia externa ou interna sem necessidade de transfusão, hematoma no local da punção, infecção localizada no local da punção, perda de pulsos ou claudicação do membro abordado, sem sinais de isquemia severa, foram consideradas complicações menores. Essas complicações menores foram avaliadas nas primeiras 24 horas após a angioplastia e antes da alta do paciente.

As informações sobre cada paciente foram registradas por um dos médicos do grupo de trabalho, em um formulário que continha os seguintes dados:

1) número de cateteres-guia, guias de ATC e número de balões utilizados em cada caso;

2) necessidade ou não de troca de material para o do outro grupo, em decorrência de dificuldade de prosseguir com o exame;

3) volume de contraste utilizado (em cc);

4) tempo de radioscopia (em minutos);

5) tempo total do exame (em minutos);

6) complicações maiores e menores, listadas anteriormente. 
Os pacientes não incluídos no estudo foram analisados em formulário anexo, em que foi especificado o motivo da exclusão.

As vias de abordagem foram escolhidas a critério do operador principal. Caso o teste de Allen fosse normal, era possível escolher a via radial; nesse caso, os materiais utilizados foram kit de micropunção $6 \mathrm{Fr}$ (Cook) e introdutor radial 6 Fr (Cook) ou Avanti (Cordis). Depois de colocado o introdutor na artéria radial direita, administravam-se $5.000 \mathrm{U}$ de heparina intravenosa. Não foram usados agentes antiespasmódicos ou vasodilatadores de forma profilática em nenhum caso.

Caso a via femoral fosse a escolhida, os materiais eram: punção com agulha Abbocath no 16 e, a seguir, colocação de introdutor Cordis ou Scimed 6 Fr na artéria femoral direita (ou esquerda se o paciente não apresentasse pulso palpável do lado direito). Foram administradas 5.000 U de heparina intra-arterial. Depois foram utilizados os mesmos cateteres diagnósti$\cos 6 \mathrm{Fr}$ utilizados na via radial.

Para ambas as vias de abordagem os materiais para ATC foram os seguintes: cateteres-guia Cordis ou Mach 1 (Boston Scientific) $6 \mathrm{Fr}$ em todos os casos, exceto para lesões de bifurcação nas quais se utilizaram cateteres $8 \mathrm{Fr}$. As guias de ATC eram Choice ou Forte (Boston Scientific), ATW ou Stabilizer (Cordis). Os balões para pré-dilatação eram Adante ou Maverick 1 ou 2 (Boston Scientific) ou U-Pass (Cordis).

Os stents implantados eram de aço inoxidável ou de liga cromo-cobalto: Bx Velocity e Bx Sonic (Cordis), Express (Boston Scientific), Lekton (Biotronik) ou Driver (Medtronic).

Uma vez terminado o procedimento, retirava-se o introdutor e comprimia-se manualmente a zona de punção inguinal até obter hemostasia, no mínimo durante 20 minutos ou por mais tempo, se houvesse persistência do sangramento. No caso de abordagem radial, deixava-se o curativo compressivo até o dia seguinte.

A única exceção para a randomização do material foi a agulha Abbocath ou a agulha de punção, utilizada uma única vez. O contraste utilizado foi iônico de alta osmolaridade (Telebrix, Guerbé) ou iônico de baixa osmolaridade (Hexabrix, Guerbé).

Após o final da ATC, o material era submetido aos seguintes passos pelo pessoal de enfermagem da sala:

1) Limpeza do material: lavagem com agentes de limpeza microbiologicamente ativos; enxágüe; secagem.

2) Esterilização: óxido de etileno; peróxido de hidrogênio.

3) Controles: esterilização; ausência de pirogênios; ausência de substâncias indutoras de hemólise.

4) Recomendações de segurança funcional: quais materiais poderão ser reutilizados; quantas vezes poderão ser reutilizados; condições em que poderão ser reutilizados.

\section{Processo de reesterilização}

Os instrumentos metálicos foram submergidos em detergente biodegradável durante vinte minutos, depois escovados e enxaguados com água sob pressão. O passo seguinte consistia na secagem com ar comprimido e na colocação em recipientes para autoclave. Os materiais plásticos e combinados também foram colocados em detergente biodegradável por vinte minutos.

Os materiais foram desmontados, enxaguados e depois montados e secos com ar comprimido. Era necessário verificar se a ponta dos materiais mantinha a forma e se conservavam o torque ou memória, sendo descartados todos os materiais que estavam em más condições.

Por último, os materiais foram embalados, esterilizados e individualizados com suas datas de validade. Os cateteres foram esterilizados com gás de óxido de etileno. Em geral, em nosso Serviço, os cateteres-guia reesterilizados são utilizados quatro ou cinco vezes e as guias e balões de angioplastia, uma ou duas vezes.

\section{Análise estatística}

Utilizou-se teste do qui-quadrado ou teste exato de Fisher, conforme as condições de aplicação, para a comparação de proporções, teste $t$ de Student para amostras independentes ou teste $U$ de Mann-Whitney para comparar as variáveis contínuas. Considerou-se significativa qualquer diferença observada nos grupos que tivesse probabilidade de ocorrência menor que 0,05. Utilizou-se o programa SPSS versão 10.01 para o processamento estatístico dos dados.

\section{Aspectos éticos}

O protocolo do ensaio foi aprovado pelo Comitê de Ética do corpo médico do Hospital de Clínicas da Faculdade de Medicina de Montevidéu, em setembro de 2001. Também foram aprovados os protótipos dos formulários de coleta de dados, de informação para o paciente, e de consentimento informado.

\section{RESULTADOS}

Foram incluídos prospectivamente e de forma randomizada todos os pacientes encaminhados para ATC no Setor de Hemodinâmica do Departamento de Cardiologia do Hospital de Clínicas entre setembro de 2001 e agosto de 2002 e entre janeiro de 2003 e dezembro de 2004, que se adequavam aos critérios de inclusão e exclusão e que assinaram o formulário de Consentimento Informado, totalizando 100 pacientes.

No total, $58 \%$ eram do sexo feminino, com média de idade de $65 \pm 8$ anos e $17 \%$ eram portadores de diabetes melito. Do total de pacientes, 16 haviam passado previamente por angioplastias e 9 haviam sido revascularizados cirurgicamente. A maioria dos pacientes era de portadores de angina instável (69\%); os demais se apresentaram com angina estável (19\%) ou 
infarto agudo do miocárdio, sendo tratados com ATC primária $(12 \%)$. A maioria $(\mathrm{n}=95)$ foi abordada por via femoral e o restante, por via radial.

Os pacientes eram portadores de lesão de 1 vaso em 68 casos, de 2 vasos em 27 casos, e de 3 vasos em 5 casos. Dentro desse universo, foram tratadas 140 lesões, sendo 97 (69\%) B2 ou C, segundo a classificação do American College of Cardiology/American Heart Association (ACC/AHA). No total, foram implantados 173 stents, sendo 48 de 2,5 mm de diâmetro $(27,7 \%), 89$ de $3 \mathrm{~mm}$ (51,4\%), 24 de $3,5 \mathrm{~mm}(13,8 \%)$, e 12 de $4 \mathrm{~mm}$ de diâmetro $(7,1 \%)$. Quanto à extensão das lesões, somente 7 tinham menos de $10 \mathrm{~mm}$ de extensão (5\%), 107 tinham entre $10 \mathrm{~mm}$ e $20 \mathrm{~mm}$ $(76,4 \%)$ e 26 tinham mais de 20 mm (18,6\%). A média de stent/lesão foi de 1,23 e de stent/paciente, de 1,73.

No total, foram realizadas 49 (49\%) ATCs com material novo (grupo 1 ) e 51 (51\%) com material reesterilizado (grupo 2). A porcentagem de lesões B2/C no grupo 1 foi de $42 / 68$ (61,8\%) e no grupo 2, de 55/72 $(61,1 \%)(p>0,99)$. O sucesso angiográfico no grupo 1 foi de $100 \%$ e no grupo 2 , de $96 \%(p=0,54)$.

A Tabela 1 apresenta os dados do procedimento e os eventos clínicos da evolução. Não foram observadas diferenças na comparação de quaisquer das variáveis relacionadas à intervenção percutânea. Em relação às complicações maiores, foram observados dois óbitos no grupo 2. No primeiro caso, o óbito decorreu de hematoma retroperitoneal com choque hipovolêmico e morte antes da cirurgia vascular. No segundo caso, o paciente foi operado, sendo constatada e reparada uma lesão da veia femoral, mas evoluiu para falência múltipla dos órgãos, falecendo 19 dias depois.
As complicações menores $\leq 24$ horas no grupo 1 ocorreram em um paciente, que apresentou hematoma no local da punção femoral e que não exigiu intervenção. No grupo 2, observamos um caso de dissecção da artéria femoral, que fechou espontaneamente, um hematoma no local da punção femoral, que não exigiu intervenção, e um caso de sangramento persistente no local da punção femoral, que cedeu com compressão prolongada.

Em relação às complicações menores $>24$ horas, no grupo 1 observou-se caso de dor persistente no local da punção radial (à ecocardiografia Doppler observou-se oclusão da artéria radial, porém houve adequado suprimento pela artéria cubital), que cedeu em poucos dias. Outro paciente apresentou quadro de febre $\left(38,5^{\circ} \mathrm{C}\right)$ durante 48 horas após a ATC, controlada com antipiréticos. No grupo 2, observou-se um caso de hematoma, que foi resolvido com tratamento conservador, e um caso de dor persistente no local da punção femoral, não vinculada à isquemia do membro abordado, que cedeu depois de alguns dias. Essas diferenças não foram estatisticamente significantes.

\section{DISCUSSÃO}

Em geral, são os países desenvolvidos que produzem a maioria dos materiais normalmente utilizados na ATC, os quais, também na maior parte dos casos, são fabricados para ser utilizados uma única vez. Essa regra baseia-se não só em questões médicas, mas também éticas, administrativas, legais e comerciais ${ }^{15}$.

O aumento do custo do sistema de saúde é um problema mundial e a Cardiologia é uma das áreas mais atingidas ${ }^{15}$. Nos países em desenvolvimento, esse impacto econômico é ainda mais forte.

TABELA 1

Dados do procedimento e os eventos clínicos da evolução

\begin{tabular}{|c|c|c|c|}
\hline Variável (por paciente) & Material novo $(n=49)$ & Material reutilizado $(n=51)$ & $\mathbf{p}$ \\
\hline Número de cateteres-guia ATC & $\begin{array}{l}1,14 \\
(1-3)\end{array}$ & $\begin{array}{l}1,33 \\
(1-2)\end{array}$ & 0,25 \\
\hline Número de guias ATC & $\begin{array}{l}1,14 \\
(1-3)\end{array}$ & $\begin{array}{l}1,22 \\
(1-2)\end{array}$ & 0,38 \\
\hline Número de balões ATC & $\begin{array}{l}0,71 \\
(0-3)\end{array}$ & $\begin{array}{l}0,55 \\
(0-3)\end{array}$ & 0,31 \\
\hline Volume de contraste (cc) & $\begin{array}{c}169 \\
(100-800)\end{array}$ & $\begin{array}{c}164 \\
(50-500)\end{array}$ & 0,84 \\
\hline Tempo de radioscopia (min) & $\begin{array}{c}14,8 \\
(1,8-33,1)\end{array}$ & $\begin{array}{c}15,4 \\
(2,0-42,8)\end{array}$ & 0,73 \\
\hline Tempo do procedimento (min) & $101,6(50-190)$ & $107,7(17-210)$ & 0,43 \\
\hline Crossover & $4(8,2 \%)$ & $8(15,7 \%)$ & 0,32 \\
\hline Complicações maiores & 0 & $2(3,9 \%)$ & 0,17 \\
\hline Complicações menores $\leq 24$ horas & $1(2,0 \%)$ & $3(5,9 \%)$ & 0,20 \\
\hline Complicações menores $>24$ horas & $2(4,1 \%)$ & $2(3,9 \%)$ & 0,88 \\
\hline
\end{tabular}


Em um mundo ideal, a tomada de decisão sobre a reutilização dos materiais deveria ser feita com base em estudos prospectivos, randomizados e cegos combinados com estudos de custos $^{15,16}$. Não encontramos dados na literatura latino-americana a esse respeito e são poucos os trabalhos em outros países que seguem essa metodologia. No Uruguai não existe regulamentação específica em relação à reutilização de materiais, sendo essa uma das causas que motivaram nosso trabalho.

Estudos observacionais apresentam discrepâncias nos resultados. Enquanto alguns registram importante economia de recursos sem aumento considerável dos riscos, outros registram que esses mesmos recursos economizados podem ser perdidos no tratamento de complicações ${ }^{8-24}$. É importante lembrar que instrumentos cirúrgicos utilizados rotineiramente na sala de operações são reutilizados de rotina, após reesterilização, sem provocar discussões éticas ou técnicas.

$\mathrm{Na}$ Cardiologia, trabalhos que avaliam a reutilização habitualmente separam os materiais usados em Eletrofisiologia dos utilizados em Cardiologia Intervencionista $^{15}$. O motivo para isso é que os cateteres utilizados nas técnicas de ablação e de estudos eletrofisiológicos diagnósticos são sólidos e, portanto, mais fáceis de reesterilizar. Na Suécia, por exemplo, é costume reutilizar marca-passos definitivos e cardiodesfibriladores implantáveis, que são removidos (caso ainda tenham suficiente vida útil) após o óbito do paciente e destinados a outros pacientes. Já na Holanda, esse procedimento é proibido por lei $i^{15-23}$.

A reutilização de cateteres de Cardiologia Intervencionista é desaconselhada por alguns, pelo fato de serem ocos e, portanto, mais difíceis de limpar e por perderem mais freqüentemente seu torque depois de reesterilizados ${ }^{17-25}$. Do ponto de vista legal, isso varia muito de um país para outro. Por exemplo, dentro da Comunidade Econômica Européia observa-se que em alguns países, como a França, a reutilização é proibida, enquanto em outros, como a Alemanha, a reutilização é autorizada se a instituição dispuser de pessoal e tecnologia adequados para o reprocessamento ${ }^{15-23}$.

Nos Estados Unidos, a reutilização só é observada nos estudos randomizados devidamente autoriza$\operatorname{dos}^{4,8}$. O trabalho de Browne et al. ${ }^{4}$ concluiu que, nos casos em que se realiza a restauração adequada de cateteres e balões de angioplastia com métodos apropriados de controle de qualidade, a reutilização pode ser segura e eficaz, com cifras de sucesso similares às dos materiais novos. Os autores sugerem a realização de estudos de custos para tomar a decisão de implementar ou não a tecnologia de reutilização com o objetivo de economizar recursos nos hospitais ${ }^{4}$.

Zubaid et al. ${ }^{24}$ relatam haver realizado o primeiro trabalho randomizado e duplo-cego sobre esse tema baseado em 377 procedimentos de ATC para comparar a eficácia e a segurança do material usado em relação ao novo. O sucesso do procedimento foi similar em ambos os grupos do estudo, assim como o volume de contraste, o tempo de radioscopia, a incidência de febre e a taxa de eventos cardíacos maiores após trinta dias. Esses autores concluem que a reutilização de cateteres e balões de ATC é tão eficiente e segura quanto a utilização de materiais novos.

O risco da reutilização inclui reação a pirogênios e infecção por bactérias ou vírus. Não há bases suficientes para se pensar na transmissão da hepatite B ou C, do HIV ou da doença de Creutzfeldt-Jakob². Apesar disso, persiste a preocupação sobre a transmissão dessas doenças. A Organização Mundial da Saúde (OMS) apresentou, em março de 1997, um relatório sobre os riscos de transmissão da doença de Creutzfeldt-Jakob e novas recomendações sobre o reprocessamento e a reesterilização com autoclave ${ }^{23}$. Outros métodos de reesterilização desses materiais, aceitos internacionalmente, são o óxido de etileno e o peróxido de hidrogênio ${ }^{22}$.

Em relação aos nossos resultados, podemos observar que não houve diferença significativa em relação ao número de cateteres, balões e guias ou ao volume de contraste e à duração geral ou tempo de radioscopia dos procedimentos. Também não houve diferença na taxa de complicações maiores ou menores, imediatas ou após 24 horas.

Assim, podemos concluir que é tão seguro e eficaz trabalhar com materiais reesterilizados quanto com materiais novos. Para alcançar esses objetivos, é imprescindível seguir estritamente todas as normas vinculadas à limpeza do material e sua correta reesterilização. Esses achados apóiam a prática de ATC com material reesterilizado em nossa população.

\section{Limitações do estudo}

Este trabalho sofreu uma interrupção temporária entre agosto de 2002 e janeiro de 2003, decorrente da falta de materiais novos em nosso Setor de Hemodinâmica, o que impediu a randomização.

O tamanho das amostras no presente estudo é relativamente baixo e, por esse motivo, diferenças entre os grupos podem não ter sido detectadas.

Não foi possível realizar uma análise de custo/ eficiência por uma série de limitações, como, por exemplo, o fato de os honorários médicos serem provenientes da atividade docente e não do número de procedimentos realizados.

Não se incluiu como complicação menor a presença de calafrios secundários à reação pirogênica, o que pode ter ocultado diferenças na segurança entre o material novo e o reesterilizado.

\section{CONCLUSÕES}

O estudo REMCLI-ATC nos permitiu concluir que a realização de ATC com material reesterilizado é se- 
gura e tecnicamente factível, já que não se observa maior número de complicações maiores ou menores na população estudada, em comparação com a utilização de materiais novos. Esses resultados apóiam o uso de materiais reesterilizados na prática da intervenção coronária percutânea nos países com recursos econômicos limitados, mas faz-se necessário avaliar maior número de pacientes para poder corroborar essa afirmação de forma definitiva.

\section{REFERÊNCIAS BIBLIOGRÁFICAS}

1. Sones FM Jr, Shirey EK, Prondfit WL. Cinecoronary arteriography. Circulation. 1959;20:773-8.

2. Judkins MP. Selective coronary arteriography. I. A percutaneous transfemoral technic. Radiology. 1967;89(5):815-24.

3. http://www.msp.gub.uy/imgnoticias/12063.xls Acesso em 28 de maio de 2008.

4. Browne KF, Maldonado R, Telatnik M, Vlietstra RE, Brenner AS. Initial experience with reuse of coronary angioplasty catheters in the United States. J Am Coll Cardiol. 1997; 30(7):1735-40.

5. Bourassa MG. The reuse of single-use balloon angioplasty catheters: is it now legitimate? Am J Cardiol. 1996;78(6): 673-4.

6. Rozenman Y, Gotsman M. Reuse of balloon catheters for coronary angioplasty. J Am Coll Cardiol. 1995;26(3):840-1.

7. Mak KH, Eisenberg MJ, Plante S, Strauss BH, Arheart KL, Topol EJ. Absence of increased in-hospital complications with reused balloon catheters. Am J Cardiol. 1996;78(6):717-9.

8. Plante S, Strauss B, Goulet G, Watson RK, Chisholm RJ. Reuse of balloon catheters for coronary angioplasty: a potential costsaving strategy? J Am Coll Cardiol. 1994;24(6):1475-81.

9. Natarajan S, Williams SV. Should percutaneous transluminal coronary angioplasty catheters be reused? J Am Coll Cardiol. $1996 ; 28(1): 112-3$.

10. Mak KH, Eisenberg MJ, Eccleston DS, Brown KJ, Ellis SG, Topol EJ. Cost-efficacy modeling of catheter reuse for percutaneous transluminal coronary angioplasty. J Am Coll Cardiol. 1996;28(1):106-11.

11. Fagih B, Eisenberg MJ. Reuse of angioplasty catheters and risk of Creutzfeldt-Jakob disease. Am Heart J. 1999;137(6): 1173-8.

12. Barbeau GR. Reuse of coronary catheters: old questions, new environment. Am Heart J. 1999;137(6):1010-1.

13. Krause G, Dziekan G, Daschner FD. Reuse of coronary angioplasty balloon catheters: yes or no? Eur Heart J. 2000; 21(3):185-9.

14. Mak KH, Eisenberg MJ, Eccleston DS, Cornhill JF, Topol EJ. Reuse of coronary angioplasty equipment: technical and clinical issues. Am Heart J. 1996;131(3):624-30.

15. Rydén L. Re-use of devices in cardiology. Proceedings from a Policy Conference at the European Heart House, 5-6 February, 1998. Eur Heart J. 1998;19(11):1628-31.

16. Vaitkus PT. Patient acceptance of reused angioplasty equipment. Am Heart J. 1997;134(1):127-30.

17. Srimahachota S, Boonyaratavej S, Udayachalerm W, Sittisuk S, Suithichaiyakul T, Sangwatanaroj S, et al. Safety and acceptable initial outcomes of reused balloon catheters for percutaneous transluminal coronary angioplasty. J Med Assoc Thai. 2000;83(12):1471-7.

18. Grimandi G, Sellal O, Grimandi F, Crochet D. Risks of reusing coronary angioplasty catheters: results of an experimental study. Cathet Cardiovasc Diagn. 1996;38(2):123-30.

19. Shaw JP, Eisenberg MJ, Azoulay A, Nguyen N. Reuse of catheters for percutaneous transluminal coronary angioplasty: effects on procedure time and clinical outcomes. Catheter Cardiovasc Interv. 1999;48(1):54-60.

20. Poole-Wilson PA. The reuse of medical devices: an ethical dilemma in a changing world. Eur Heart J. 1999;Suppl G:G5-7.

21. Bertrand ME, Van Belle E. Reuse of catheters in the catheterization laboratory. Eur Heart J. 1999;Suppl G:G20-4.

22. Nottebrock D. Requirements for reuse of diagnostic and intervention catheters in cardiology. Eur Heart J. 1999;Suppl G:G25-7.

23. Howard AJ, Dedrick VA. Legal and responsibility aspects of the reuse of medical devices. Eur Heart J. 1999;Suppl $\mathrm{G}: \mathrm{G} 2-4$.

24. Zubaid M, Thomas CS, Salman H, Al-Rashdan I, Hayat N, Habashi A, et al. A randomized study of the safety and efficacy of reused angioplasty balloon catheters. Indian Heart J. $2001 ; 53(2): 167-71$. 